\title{
State of mind: Classification through self-reported affect and word use in speech.
}

\author{
Eva-Maria Rathner ${ }^{1}$, Yannik Terhorst ${ }^{1}$, Nicholas Cummins ${ }^{2}$, Björn Schuller ${ }^{2,3}$, Harald Baumeister ${ }^{1}$, \\ ${ }^{1}$ Clinical Psychology and Psychotherapy, University of Ulm, Germany \\ ${ }^{2}$ ZD.B Chair of Embedded Intelligence for Health Care and Wellbeing, University of Augsburg, Germany \\ ${ }^{3}$ GLAM -- Group on Language, Audio \& Music, Imperial College London, UK \\ eva-maria.rathnerduni-ulm.de
}

\begin{abstract}
Human state-of-mind (SOM; e.g.: perception, cognition, attention) constantly shifts due to internal and external demands. Mental health is influenced by the habitual use of either adaptive or maladaptive SOM. Therefore, the training of conscious regulation of SOM could be promising in self-help (e- and m-health), blended care and psychotherapy. The presented study indicates that SOM can be influenced by telling personal narratives. Furthermore, SOM and narrative sentiment (positive vs. negative) can be predicted through word use. Such results lay the groundwork for the development of applications that analyse text and speech for: i) the early detection of mental health; ii) the early detection of maladaptive changes in emotion dynamics; (iii) the use of personal narratives to improve emotion regulation skills; iv) the distribution of tailored interventions; and finally, v) the evaluation of therapy outcome.

Index Terms: self-reported affect, language use, positive narrative, negative narrative, Linguistic Inquiry Word Count
\end{abstract}

\section{Introduction}

Continuous and adaptive regulation of SOM is pivotal for mental functioning and behaviour regulation [1]. One key aspect of the human experience are our emotions, as they affect our attention [2], perception [3], cognition [4] decision making [5], judgement [6], memory storage [3], memory retrieval [7] and regulate behaviour [8].

According to Russell's theory of a core affect (CA) [9], every emotional state is a combined value of valence and arousal. Valence represents the hedonistic continuum from negative to positive, while arousal reflects the physical agitation component of emotional experience. These aspects of the CA follow the general principle of mood congruency; i.e., a positive CA shifts attention to positive material while a negative CA to negative material [6]. Furthermore, high arousal facilitates access to high-arousal stimuli and vice-versa [2].

In an embodiment approach to cognition (see [7]) this bidirectional relationship can be used to willingly influence CA and, furthermore, all previously mentioned aspects of SOM. Although the majority of individuals state that they are at the mercy of their emotions, there is a strong body of evidence that indicates that emotions regulation can be trained [10]. Maladaptive emotion regulation is associated with diminished well-being [1], interpersonal problems [10], psychiatric conditions [1], and overall morbidity [11].

Psychotherapy is a method to change ones SOM through the use of language and a corrective therapeutical relationship. To date, there is proof of both common (e.g. relationship) and specific (e.g. used methods, setting, etc.) factors of change that result in a therapy outcome [12]. Depending on the exact theoretical conceptualisation about human nature, diverse therapy schools (e.g. behaviour therapy, psychoanalysis, and systemic therapy) state diverging specific factors of change [12]. Conventional therapeutic approaches focused on the recall of past and negative narratives (NN) about oneself, while more modern therapy schools focus on the presence, the future and the development of skills and resources through the construction of positive narratives (PN) about oneself.

The following study explores if CA can be influenced by the personal narratives we tell ourselves and others. This principle could be used in e- and m-health intervention or in the classical psychotherapeutic setting to enhance individuals' emotion regulation abilities. Earlier research in this regard has mainly focused on written language and it's positive effect on health and well-being [13] . Word use has been associated with personality [14], academic outcome [15], longevity [16] and psychopathology [17]. In regards to SOM, the LIWC categories of interest in the above-mentioned literature are positive emotion words, negative emotion words, social words and selfreferencing words. Positive emotion and social word use is correlated with longevity [16] and health [13]. Negative emotion and self-referencing word use on the other hand, is associated with depression [17], suicide and neuroticism [14].

From a neurological perspective, the brain is constantly regulating behaviour and behaviour in turn shapes the brain. This dynamic interaction is the neural basis of emotion, cognition and plasticity, or in other words SOM [18]. Individual differences in neuroanatomy affect perceptual and cognitive abilities. Studies could show that psychotherapy alters brain structure [19]. Based on these assumptions, it is possible that the habitual use of PN could lead to more time spent in functional SOM through altering brain structure and vice versa. In the same manner, SOM, operationalized via CA could be predicted through word use. When focusing on valence and arousal the potential biological pathways that moderate the correlation between morbidity and affect [20] could be illuminated. Possible pathways could be the cardiovascular system [21] and the immune system [22].

This study aims to provide the groundwork for the detection of PN and NN through word use in speech. Subsequently, transcribed therapy protocols could be analysed and the amount of time spent in PN and NN associated with a therapy outcome. Using the Ulm State-of-Mind in Speech (USoMS) corpus, we herein explore three research questions: 1) that the reporting of a PN or NN affects SOM operationalized via $\mathrm{CA} ; 2$ ) that word analysis can predict the category of a narrative (PN vs. NN); and, 3) that SOM can be predicted by word analysis. 


\section{Methods}

The study took place at the Department of Clinical Psychology and Psychotherapy, at the University of Ulm and was approved by the local Ethics Committee. Data was collected in two waves in June/July and November/December 2017. Participants were recruited online and were reimbursed for their time. Parts of this dataset have been released for the Interspeech 2018 challenge named as Ulm State-of-Mind in Speech (USoMS) [23].

A total of 127 speakers were recorded. Audio was captured in Stereo, converted to mono, at $44.1 \mathrm{kHz}, 32 \mathrm{bit}$, and manually cleaned. Three speakers had to be excluded due to technical failure. One participant withdrew from the study due to personal reasons. Therefore, in total 123 recordings have been manually transcribed.

All speakers gave written consent to participate in the study and were seated in front of a white wall facing the interviewer and a camera. At first, they reported their age, gender, educational level and mother tongue. Mean age was $\mathrm{m}=22.43$ (sd =3.66). All participants had German language skills on native speaker level. The demographic characteristics of the sample are presented in Table 1.

\subsection{Procedure}

Data collection started with a baseline assessment of CA (t0) on a 10 point likert scale for both arousal and valence. As emotions fluctuate over time due to internal and external demands [24], [1], the participants reported their CA pre and post every spoken narrative. We assessed two positive and two negative narratives, each approximately 5 minutes. The introduction for negative narratives was: "Please remember a time in your life when you were facing a seemingly unsolvable problem and report as detailed as possible over the next five minutes". The wording of the positive narrative task was: "Please report of a time in your life were you found a solution, where you felt powerful, happy and content. Describe that story in-depth over the next five minutes". The participants were given no preparation or rehearsal time.

In summary, the study protocol was as followed: t0 affect, negative narrative1, t1 affect, negative narrative2, t 2 affect, positive narrative $1, \mathrm{t} 3$ affect, positive narrative $2, \mathrm{t} 4$ affect.

\subsection{Self-reported affect}

Russel's model of a CA [9] states that every emotional experience can be categorized on the scales valence (negativepositive) and arousal (enervated-energized). This model's advantage lies clearly the universality; it is not language or culture bound. CA can be used to assess conscious emotional states via self-report once or over a certain period of time [25]. Repeated measurements of emotions are crucial because emotions nature is to change over time [1]. Means and standard deviations of CA in the sample on all measurement points can be seen in Table 2.

\subsection{Word Analysis}

Linguistic Inquiry Word Count (LIWC, [26]) is a widely used software in psychology and it is available for German language in the version 1.0 [27]; it has been widely used in speech analyses for tals such as depression [28] and autism detection [29]. It checks each word of a document against a predefined dictionary of more than 2.300 words and word stems. Each word is assigned to linguistic categories and the percentage of total words in each category is reported. For example, the word
Table 1: Demographic characteristics of the sample.

\begin{tabular}{ccc}
\hline Variable & Label & Label \\
\hline \multirow{2}{*}{ Gender } & Male & Female \\
& $\mathrm{N}=18(14.63 \%)$ & $\mathrm{N}=105(85.37 \%)$ \\
Age & Male & Female \\
Education & $\mathrm{M}=22.71, \mathrm{sd}=2.78)$ & $\mathrm{M}=22.38,(\mathrm{sd}=3.75)$ \\
level & A-level & Bachelor degree \\
Language & German & $28(22.58 \%)$ \\
skills & $113(92.62 \%)$ & Bilingual \\
& & $9(7.38 \%)$ \\
\hline
\end{tabular}

Table 2: Means and standard deviations of valence and arousal.

\begin{tabular}{ccc}
\hline $\begin{array}{c}\text { Measurement } \\
\text { point }\end{array}$ & Valence & Arousal \\
\hline 0 & $\mathrm{M}=6.94(\mathrm{sd}=1.48)$ & $\mathrm{M}=5.62(\mathrm{sd}=1.54)$ \\
1 & $\mathrm{M}=5.83(\mathrm{sd}=1.69)$ & $\mathrm{M}=6.60(\mathrm{sd}=1.37)$ \\
2 & $\mathrm{M}=5.70(\mathrm{sd}=1.74)$ & $\mathrm{M}=6.50(\mathrm{sd}=1.47)$ \\
3 & $\mathrm{M}=7.50(\mathrm{sd}=1.10)$ & $\mathrm{M}=6.82(\mathrm{sd}=1.35)$ \\
4 & $\mathrm{M}=7.89(\mathrm{sd}=1.14)$ & $\mathrm{M}=6.92(\mathrm{sd}=1.32)$ \\
\hline
\end{tabular}

Table 3: Means and standard deviations of characteristics of general word use.

\begin{tabular}{lll}
\hline Category & Positive Narrative & Negative Narrative \\
\hline self & $\mathrm{M}=14.09(\mathrm{sd}=4.19)$ & $\mathrm{M}=15.34(\mathrm{sd}=4.24)$ \\
positive & $\mathrm{M}=5.98(\mathrm{sd}=1.66)$ & $\mathrm{M}=3.58(\mathrm{sd}=1.12)$ \\
negative & $\mathrm{M}=1.37(\mathrm{sd}=0.77)$ & $\mathrm{M}=3.42(\mathrm{sd}=1.42)$ \\
social & $\mathrm{M}=1.57(\mathrm{sd}=1.07)$ & $\mathrm{M}=1.58(\mathrm{sd}=1.10)$ \\
\hline
\end{tabular}

smile falls into four categories: happiness, positive emotion, cognitive processing, social orientation and psychological distancing. Our analysis focused on four linguistic indicators: i) self-referencing words; ii) social words; iii) positive emotion words; and, iv) negative emotion words. There is a vast body of evidence on the influence of those four categories on mental and physical health (see the introduction section for detailed description) [14], [16], [17], [13]. In the following statistical analyses, the categories self-referencing, positive emotional, negative emotional and social words were used. The descriptive characteristics of general word use are summarized in Table 3.

\subsection{Statistical Analyses}

Before being our analysis all speech recordings were manually transcribed. For the analysis it was assumed, that measurement points (Level 1) of a person are nested within a person (Level 2). Multilevel models (MLM) were then used to represent this hierarchical structure. For the multivariate analyses we followed the procedure described by Goldstein [30] was applied (see [31] for further introduction).

To test Research Question 1, a multivariate MLM with random intercept and random slope was defined (herein referred to as Model 1). The random intercepts and slopes were assumed to represent the variance across individuals. The valence and arousal scores were z-standardized. The category of narrative (positive or negative) was used as an effect coded (positive 1, negative 0) predictor and restricted maximum likelihood was used as the estimator.

For Research Question 2, logistic MLM with random intercept and random slopes were used (herein referred to as Model $2 a$ and $2 b$ ). Random intercepts and slopes were assumed to represent variations across individuals. The narrative category was dummy-coded (negative: 0 , positive: 1 ) and used 
as the outcome variable. Percentages of word use for the categories: self-referencing, positive emotional, negative emotional and social words were used as the predictors, and maximum likelihood with Lapalce approximation was used as the estimator.

Research Question 3, was tested analogous to Research Question 1, with the exception that the LIWC word categories were used as predictors. Analyses for Research Question 1 and 3 were conducted in Statistical Package for the Social Sciences (SPSS) Version 24 [32]. Logistic MLM was carried out using $\mathrm{R}$ [33] and the packages lme4 [34] and lmerTest.[35].

\section{Results and Discussion}

\subsection{Reporting a personal narrative is affecting state of mind}

Model 1 was used to assess the effect of narratives on CA. The intraclass correlations (ICCs) indicate the suitability of using MLM $\left(\mathrm{ICC}_{\text {valence }}=.33, \mathrm{ICC}_{\text {arousal }}=.72\right)$. The intercepts of valence and arousal are zero $(\mathrm{p}=.944, \mathrm{p}=.946)$. Results of the statistical analysis indicates that valence and arousal are indeed influenced by narrative (negative or positive) with a fixed estimate of .61 (95\% CI: .53 to .69) and beta $=.10$ (95\% CI: .05 to .15). Interestingly, the influence on valence and arousal appears to vary across individuals $\left(\right.$ var $_{\text {valence }}=.14,95 \% \mathrm{CI}$ : .10 to .21 ; vararousal $=.03,95 \% \mathrm{CI}: .01$ to .06$)$. The intercepts of valence and arousal are correlated $\mathrm{r}=.38,95 \% \mathrm{CI}$ : .20 to .54 ). Further, the intercepts of valence $(\mathrm{r}=-.54,95 \% \mathrm{CI}:-.69$ to $.34)$ and arousal $(\mathrm{r}=.33,95 \% \mathrm{CI}: .12$ to .52$)$ are correlated with the influence of the narrative on valence. No other significant correlations were observed. An overview of the full analysis is given in Table 4 .

These findings indicate that individuals can influence their SOM/CA simply by telling a personal PN or NN. Arousal and valence were correlated and varied across individuals, implicating that there are inter-individual differences in the effect of storytelling on CA. While arousal rose after telling personal stories, valence decreased after telling $\mathrm{NN}$ and increased after telling PN. Arousal was correlated positive with the influence of narrative on valence, valence was correlated negative.

By showing, that a simple practice like telling a PN can positively influence SOM/CA numerous applications could be tested. Given the implications of positive and negative affect on mental health, physical health, and psychosocial functioning [22] it is very promising to use personal story telling as a therapeutic tool (e.g.: in e- and $\mathrm{m}$-health interventions) to improve individual health and quality of life.

\subsection{Sentiment of narrative (PN vs. $\mathrm{NN}$ ) can be predicted through word use}

Model $2 \mathrm{a}$ and $2 \mathrm{~b}$ test whether sentiment of narrative can be predicted trough word use in personal narratives. The initial logistic MLM achieved no convergence. However, convergence was achieved if only the intercept and the influence of positive emotional words (Model 2a), or the intercept and the influence of negative emotional words (Model $2 \mathrm{~b})$ were defined as random slopes. The likelihood ratio tests for both models indicate, that random slopes of positive and negative emotional words yield significant better fit (model 2a: $\mathrm{D}=55.67, \mathrm{df}=2, \mathrm{p}<.001 ;$ model $2 \mathrm{~b}: \mathrm{D}=63.06, \mathrm{df}=2$, $\mathrm{p}<.001)$.
Table 4: Output of the multivariate MLM on the influence on narratives on SOM.

\begin{tabular}{|c|c|c|c|}
\hline & Estimate & $\begin{array}{l}\text { Standard } \\
\text { error }\end{array}$ & $P$ \\
\hline \multicolumn{4}{|l|}{ Modelfit } \\
\hline Restricted -2LL ${ }^{1}$ & 2070.515 & & \\
\hline $\mathrm{AIC}^{2}$ & 2092.515 & & \\
\hline $\mathrm{BIC}^{3}$ & 2146.345 & & \\
\hline \multicolumn{4}{|l|}{ Fixed Part } \\
\hline Intercept ${ }_{v a l e n c e}$ & .005 & .064 & .944 \\
\hline Narrative $_{\text {valence }}$ & .613 & .040 & $<.001$ \\
\hline Intercept $t_{\text {arousal }}$ & -.005 & .069 & .946 \\
\hline Narrative ${ }_{\text {arousal }}$ & .099 & .065 & $<.001$ \\
\hline \multicolumn{4}{|l|}{ Random Part } \\
\hline$\sigma^{2}$ Intercept(valence) & .451 & .065 & $<.001$ \\
\hline$\sigma^{2}$ Narrative(valence) & .144 & .026 & $<.001$ \\
\hline$\sigma^{2}$ Intercept(arousal) & .528 & .075 & $<.001$ \\
\hline$\sigma^{2}$ Narrative(arousal) & .028 & .012 & .017 \\
\hline$\sigma^{2}$ Residuum & .236 & .015 & $<.001$ \\
\hline $\operatorname{Cor}_{(1,2)}$ & -.541 & .089 & $<.001$ \\
\hline $\operatorname{Cor}_{(1,3)}$ & .380 & .088 & $<.001$ \\
\hline $\operatorname{Cor}_{(1,4)}$ & -.085 & .169 & .615 \\
\hline $\operatorname{Cor}_{(2,3)}$ & .334 & .103 & .001 \\
\hline $\operatorname{Cor}_{(2,4)}$ & .161 & .188 & .392 \\
\hline $\operatorname{Cor}_{(3,4)}$ & -.093 & .167 & .577 \\
\hline
\end{tabular}

Note: 1) log likelihood 2) Akaike information criterion, 3) Bayesian information criterion

Model $2 \mathrm{a}$ showed, that self-referencing words (Odds ratio $=.74, \mathrm{p}<.001$ ) and negative emotional words (Odds ratio $=.06, \mathrm{p}<.001)$ decrease the odds for a positive narrative. Vice versa was also observed, positive emotion words increase the odds for a positive narrative (Odds ratio $=32.35, \mathrm{p}<.001$ ). Our analysis indicates that social words have no influence $(p=.941)$. The variance of intercepts was $v a r=161.11$ and of positive emotional words var $=7.24$. The correlation between intercept and positive emotional words was $r=-.94$

Model $2 \mathrm{~b}$ on the other hand indicates that self-referencing words (Odds ratio $=.80, \mathrm{p}<.001$ ) and negative emotional words (Odds ratio $=.03, \mathrm{p}<.001$ ) increase the odds for a negative narrative, while positive emotional words reduce the odds (Odds ratio $=7.10, \mathrm{p}<.001$ ). Social words had no influence $(\mathrm{p}=.869)$. The variance of intercepts was var $=41.19$ and of negative emotional words var $=7.225$. The correlation between intercept and emotional words was $\mathrm{r}=-.89$.

A systematic review shows that psychotherapy alters brain structure [19]. One effect of psychotherapy is the change of a persons' default SOM. In line with that, the habitual use of a PN could lead to more time spent in functional SOM through altering brain structure and vice versa. Furthermore, an automatized use of a positive SOM could lead to a more adaptive selection of attention, functional cognition about oneself and others, and enhanced behaviour regulation abilities.

\subsection{SOM can be predicted through word use}

Our multivariate MLM using word categories as predictors resulted in a non-positive definite Hessian matrix. Univariate MLM was used instead. MLM for valence showed that valence 
Table 5: Output of the multivariate MLM for Research Question 3.

\begin{tabular}{|c|c|c|c|}
\hline & Estimate & $\begin{array}{l}\text { Standard } \\
\text { error }\end{array}$ & $P$ \\
\hline \multicolumn{4}{|l|}{ Modelfit } \\
\hline Restricted $-2 \mathrm{LL}^{I}$ & 164.599 & & \\
\hline $\mathrm{AIC}^{2}$ & 176.599 & & \\
\hline $\mathrm{BIC}^{3}$ & 205.955 & & \\
\hline \multicolumn{4}{|l|}{ Fixed Part } \\
\hline Intercept & -.205 & .314 & .515 \\
\hline Intercept $_{\text {self }}$ & -.008 & .015 & .586 \\
\hline Narrative $_{\text {positive }}$ & .226 & .041 & $<.001$ \\
\hline Interceptnegative & -.167 & .052 & .002 \\
\hline Narrative $_{\text {social }}$ & -.159 & .127 & .216 \\
\hline \multicolumn{4}{|l|}{ Random Part } \\
\hline$\sigma^{2}$ Intercept & 8.450 & 1.335 & $<.001$ \\
\hline$\sigma^{2}$ Intercept(self) & .020 & .003 & $<.001$ \\
\hline$\sigma^{2}$ Intercept(positive) & .162 & .024 & $<.001$ \\
\hline$\sigma^{2}$ Intercept(negative) & .250 & .039 & $<.001$ \\
\hline$\sigma^{2}$ Intercept(social) & 1.832 & .258 & $<.001$ \\
\hline$\sigma^{2}$ Residuum & $<.001$ & $<.001$ & $<.001$ \\
\hline
\end{tabular}

is influenced by positive emotional words (beta $=.22,95 \% \mathrm{CI}$ : .15 to .31 ) and negative emotional words (beta $=-.17,95 \%$ CI: -.27 to -.06 ). On the other hand, social words (beta $=-.16$, $95 \%$ CI: -.41 to .09 ) and self-referencing words (beta $=-.01$, $95 \%$ CI: -.04 to .02) had no significant influence. The analysis indicates that individuals vary in their use of self-referencing words (var $=.02,95 \%$ CI: .01 to .03 ), social words ( $\operatorname{var}=1.83$, $95 \%$ CI: 1.39 to 2.42 ), positive emotional words (var $=.16$, $95 \%$ CI: .12 to .22 ) and negative emotional words (var $=.25$, $95 \%$ CI: .18 to .34). The univariate MLM for arousal achieved no convergence. An overview of the full analysis is given in Table 5 .

These models show, that only one parameter of CA, namely valence, was associated with type of narrative. There are some explanations for this phenomenon: Firstly, arousal rose after telling a narrative no matter if it was positive or negative. Connecting with yourself through expressing yourself over narratives might lead to an altered perception of physiological parameters like arousal. Secondly, the change in arousal and the variance of arousal was rather small compared to valence. Thirdly, the word categories we choose for our models (social words, positive emotion words, negative emotion words, selfreferencing words) might fit valence better than arousal. In further studies other LIWC categories could be used to predict arousal (e.g.: perception, feelings, body, etc.). We choose the earlier mentioned categories due to their implications on overall psychological functioning and health outcomes [13], [14], [16], [17]. Comparison of general or emotion specific dictionaries show that the use of the emotion categories of the LIWC is sufficiently comparable to the use of general dictionaries [42].

Texts produced while using the interventions could be used to gain insight into an individual's SOM. Not only the detection of actual SOM is crucial for therapeutic outcome, also the changes in SOM over time is an important source of information about an individual's health. Most affective disorders are characterized by changed emotion dynamics.
Emotion dynamics, (e.g. inertia, variability, instability) have been associated with the onset of depression [36] and overall psychopathology [1]. Therefore, automatized detection of SOM and its dynamics could be used to: i) detect the onset of a psychiatric condition early; ii) pre-screen individuals in a stepped care-approach to deliver tailored-interventions (e.g.: standard care, blended care, e-health, etc.); and finally, iii) evaluate therapy outcome. The early detection of psychiatric conditions is associated with course and treatment outcomes as well as a reduced personal and societal burden [37]. A stepped care approach would reduce costs for health care providers by pre-selecting patients according to their needs, reduce emotional burden in individuals caused by long waiting periods for treatment, and help foster patient empowerment [38].

Recently there is considerable research interest in automated recognition of affect from speech or written language focusing on nearly real-time recognition of emotional states [39][40]. Such close to real-time results could be used as feedback in a therapeutic embodiment approach to assist individuals in learning to regulate emotion willingly. In line with that, individuals could be guided to report PN on a regular basis and therefore learn to influence their affect willingly. For example, the use of $\mathrm{PN}$ in e- and m-health interventions applications that detect affect through speech in nearly realtime and give visual feedback could provide an easy useable bio-feedback-training. In embodiment research it has been shown that maintaining specific body positions can influence SOM as well as physiological parameters [41]. If this is functioning with body postures, this might also work with the modulation of speech. Individuals could learn to change their emotion by regulating their voice and modifying their personal narratives to maintain or achieve health and well-being.

\section{Conclusion}

The current study gives insight into the effects of reporting a personal narrative on SOM. We observed that the reporting of negative narratives (NN) leads to more negative valence and a minimal rise in arousal, while reporting a positive narrative $(\mathrm{PN})$ is related to elevated positive valence and a small rise in arousal. This effect might be used to foster an individual's emotion regulation skills over the habitual use of PN. Furthermore, the sentiment of narrative could be predicted through word use alone. In a neuroplasticity approach this correlation could be used to analyse the time spent in PN or NN and relate them to health and therapy outcomes. Finally, we also observed that valence could be predicted through word use, giving the opportunity to monitor individuals affect over word analysis. If these findings could be replicated with deep machine learning, they could contribute to the development of applications that analyse text and speech for automatized assessment of mental health, detection of emotion dynamics, the use of personal narratives to improve emotion regulation skills in self-help, blended-care and therapy and finally the evaluation of therapy outcome.

\section{Acknowledgements}

This research has also received funding from the Innovative Medicines Initiative 2 Joint Undertaking under grant agreement No 115902. This Joint Undertaking receives support from the European Union's Horizon 2020 research and innovation programme and EFPIA. The authors would like to thank Alice Baird for the technical advice, Jana Mönnekes, Sonya Goosen and Sandy Spormann for transcribing. 


\section{References}

[1] M. Houben, W. Van Den Noortgate, and P. Kuppens, "The relation between short term emotion dynamics and psychological well-being: A meta-analysis.," Psychol. Bull., vol. 141, no. 4, pp. 901-930, 2015.

[2] S. Shapiro, D. J. MacInnis, and C. W. Park, "Understanding program-induced mood effects: Decoupling arousal from valence," J. Advert., vol. 31, no. 4, pp. 15-26, 2002.

[3] M. Mather and M. R. Sutherland, "Arousal-biased competition in perception and memory," Perspect. Psychol. Sci., vol. 6, no. 2, pp. 114-133, 2011.

[4] H. Leventhal and K. Scherer, "The relationship of emotion to cognition: A functional approach to a semantic controversy," Cogn. Emot., vol. 1, no. 1, pp. 3-28, 1987.

[5] E. Peters, D. Västfjäll, T. Gärling, and P. Slovic, "Affect and decision making: A 'hot' topic," J. Behav. Decis. Mak., vol. 19 , no. 2, pp. 79-85, 2006.

[6] N. Schwarz and G. L. Clore, "Mood, misattribution, and judgements of well-being: Informative and directive functions of affective states," J. Pers. Soc. Psychol., vol. 45, no. 3, pp. 513-523, 1983

[7] P. M. Niedenthal, L. W. Barsalou, P. Winkielman, S. KrauthGruber, and F. Ric, "Embodiment in attitudes, social perception, and emotion," Personal. Soc. Psychol. Rev., vol. 9, no. 3, pp. 184-211, 2005.

[8] B. Hommel, A. Moors, D. Sander, and J. Deonna, "Emotion meets action: Towards an integration of research and theory,' Emot. Rev., vol. 9, no. 4, pp. 295-298, 2017

[9] J. A. Russell, "Core affect and the psychological construction of emotion," Psychol. Rev., vol. 110, no. 1, pp. 145-172, 2003

[10] J. P. Mulki, F. Jaramillo, E. A. Goad, and M. R. Pesquera, "Regulation of emotions, interpersonal conflict, and job performance for sales people," J. Bus. Res., vol. 68, no. 3, pp. 623-630, 2015.

[11] G. Sheppes, G. Suri, and J. J. Gross, "Emotion regulation and psychopathology," Annu. Rev. Clin. Psychol., vol. 11, pp. 379-405, 2015

[12] S. E. Stevens, M. T. Hynan, and M. Allen, "A meta-analysis of common factor and specific treatment effects across the outcome domains of the phase model of psychotherapy," Clin. Psychol. Sci. Pract., vol. 7, pp. 273-290, 2000.

[13] J. W. Pennebaker and J. D. Seagal, "Forming a story: The health benefits of narrative," J. Clin. Psychol., vol. 55, no. 10, pp. 1243-1254, 1999.

[14] T. Holtgraves, "Text messaging, personality, and the social context," J. Res. Pers., vol. 45, pp. 92-99, 2011.

[15] R. L. Robinson, R. Navea, and W. Ickes, "Predicting final course performance from students' written self-introductions: A LIWC analysis," J. Lang. Soc. Psychol., vol. 32, no. 4, pp. $469-479,2013$

[16] S. D. Pressman and S. Cohen, "Positive emotion word use and longevity in famous deceased psychologists," Heal. Psychol., vol. 31, no. 3, pp. 297-305, 2012.

[17] A. J. Rodriguez, S. E. Holleran, and M. R. Mehl, "Reading between the lines: The lay assessment of subclinical depression from written self-descriptions," J. Pers., vol. 78, no. 2, pp. 575-598, 2010 .

[18] R. J. Zatorre, R. . Fields, and H. Johansen-Berg, "Plasticity in Gray and White: Neuroimaging changes in brain structure during learning," Nat. Neurosci., vol. 15, no. 4, pp. 528-536, 2013.

[19] A. Barsaglini, G. Sartori, S. Benetti, W. Pettersson-Yeo, and A. Mechelli, "The effects of psychotherapy on brain function: A systematic and critical review," Prog. Neurobiol., vol. 114, pp. 1-4, 2014.

[20] S. Cohen and S. D. Pressman, "Positive affect and health," Psychol. Sci., vol. 15, no. 3, pp. 122-125, 2006.

[21] A. R. Schwerdtfeger, H. Scharnagl, T. Stojakovic, and E.-M Rathner, "Cognitive avoidant coping is associated with higher carotid intima media thickness among middle-aged adults," Int. J. Behav. Med., vol. 22, no. 5, pp. 597-604, 2015.

[22] A. L. Marsland, S. Cohen, B. S. Rabin, and S. B. Manuck, "Trait positive affect and antibody response to hepatitis B vaccination," Brain. Behav. Immun., vol. 20, no. 3, pp. 261269, 2006.

[23] B. Schuller et al., "The INTERSPEECH 2018 Computational Paralinguistics Challenge: Atypical \& self-assessed affect, crying \& heart beats," Available: http://emotionresearch.net/sigs/speech-sig/is2018_compare.pdf

[24] P. Koval, P. Kuppens, N. B. Allen, and L. Sheeber, "Getting stuck in depression: The roles of rumination and emotional inertia," Cogn. Emot., vol. 26, no. 8, pp. 1412-1427, 2012.

[25] L. F. Barrett and J. A. Russell, "The structure of current affect: Controversies and emerging consensus," Curr. Dir. Psychol. Sci., vol. 8, no. 1, pp. 10-14, 1999.

[26] J. W. Pennebaker, R. J. Booth, and M. E. Francis, Operator's Manual: Linguistic Inquiry and Word Count - LIWC2007. Austin, Texas: LIWC.net, 2007.

[27] J. W. Pennebaker, R. J. Booth, R. L. Boyd, and M. E. Francis, "Linguistic Inquiry and Word Count." Erlbaum Publishers, Mahwah, NJ, USA, 2015.

[28] Y. Gong and C. Poellabauer, "Topic modeling based multimodal depression detection," in Proceedings of the 7th Annual Workshop on Audio/Visual Emotion Challenge, 2017, pp. 69-76.

[29] M. Kumar, R. Gupta, D. Bone, N. Malandrakis, S. Bishop, and S. . Narayanan, "Objective language feature analysis in children with neurodevelopmental disorders during autism assessment," in Proceedings INTERSPEECH 2016, 17th Annual Conference of the International Speech Communication Association, 2016, pp. 2721-2725.

[30] H. Goldstein, Multi-level statistical models. NY, USA: Halsted, 1995

[31] R. C. MacCallum, K. Cheongtan, and W. B. Malarkey, "Studying multivariate change using multilevel models and latent curve models," Multivar. Behav Res, vol. 32, no. 3, pp. 215-253, 1997.

[32] IBM Corp., "IBM SPSS Advanced Statistics." IBM Corp., Armonk, NY, USA, 2016.

[33] R Core Team, R: A Language and environment for statistical computing. Vienna, Austria: R Foundation for Statistical Computing, 2017.

[34] D. Bates, M. Mächler, B. Bolker, and S. Walker, "Fitting linear mixed-effects models using lme4," J. Stat. Softw., vol. 67, pp. 1-48, 2015.

[35] A. Kuznetsova, P. B. Brockhoff, and R. H. B. Christensen, "ImerTest package: Tests in linear mixed effects models," $J$. Stat. Softw., vol. 82, pp. 1-26, 2017.

[36] P. Koval, M. L. Pe, K. Meers, and P. Kuppens, "Affect dynamics in relation to depressive symptoms: Variable, unstable or inert?," Emotion, vol. 13, no. 6, pp. 1132-1141, 2013.

[37] H. Baumeister, J. Lin, and D. D. Ebert, "Internet- und mobilebasierte Ansaetze," Bundesgesundheitsblatt. Gesundheitsforschung. Gesundheitsschutz, vol. 60, no. 4, pp. 436-444, 2017.

[38] D. Bakker, N. Kazantzis, D. Rickwood, and N. Rickard, "Mental health smartphone apps: Review and evidence-based recommendations for future developments," JMIR Ment. Heal., vol. 3, no. 1, p. e7, 2016.

[39] E. Marchi, F. Eyben, G. Hagerer, and B. Schuller, "Real-time tracking of speakers' emotions, states, and traits on mobile platforms," in Proceedings INTERSPEECH 2016, 17th Annual Conference of the International Speech Communication Association, 2016, pp. 1182-1183.

[40] A. Tsiartas et al., "Sensay analyticstm: A real-time speakerstate platform," in 2017 IEEE International Conference on Acoustics, Speech and Signal Processing (ICASSP), 2017. pp. 6582-6483.

[41] J. Cesario and M. M. McDonald, "Bodies in context: Power poses as a computation of action possibility," Soc. Cogn., vol. 31, no. 2, pp. 260-274, 2013. 